\section{A model of a}

quasicrystal structure.

to three dimen-

sions produced

Steinhardt's long-

imagined icosahedral quasicrystal.

Unknown to them, a couple of hundred kilometres away, a materials scientist had obtained a diffraction pattern unlike any he had seen before - for a rapidly cooled alloy of aluminium and manganese. The researcher was Dan Shechtman, and he was working at the National Bureau of Standards (now the National Institute of Standards and Technology) in Gaithersburg, Maryland. The diffraction pattern had ten-fold rotational symmetry. Astonished, Shechtman drew its concentric rings of ten dots in his lab book, writing "10-fold???” next to it. He didn't know it yet, but he had just discovered the first quasicrystal. In 2011, he won the Nobel Prize in Chemistry for it.

Steinhardt and Levine, recognizing the collective implications, published a paper on their theory in Physical Review Letters, laying the foundations of the field of quasicrystal research (D. Levine and P. J. Steinhardt Phys. Rev. Lett. 53, 2477; 1984).

Hundreds of quasicrystals with different symmetries have now been made in the lab. Non-stick frying pans were among the first applications of quasicrystals, owing to the alloys' low friction, high hardness and low surface reactivity. Steel hardened by small quasicrystal particles is used in needles for acupuncture and surgery, dental instruments and razor blades. Quasicrystals have been discovered in materials other than metals, including polymers and mixtures of nanoparticles. Computer simulations suggest that quasicrystals should be even more ubiquitous.

All these quasicrystals were humanmade, but Steinhardt became curious about naturally occurring ones. In 1999, now on the faculty at Princeton University in New Jersey, he set out on a quest that few theoretical physicists could imagine - to find one. This is the true heart of his book.

\section{GLOBAL HUNT}

Steinhardt started by looking to the past - in museums, which have huge collections of mineral samples from around the world. Perhaps, he surmised, one of these was a misidentified quasicrystal. $\mathrm{He}$ assembled a crack team of fellow hopefuls — including a geoscientist, an electron microscopist and an undergraduate - and the hunt began. Years of combing through dusty archives turned up nothing, until a box from the Museum of Natural History in Florence, Italy, arrived at Princeton containing a nearly invisible speck of a rare mineral, khatyrkite. On 2 January 2009 , the researchers became certain that they had discovered a natural quasicrystal (later named icosahedrite). But where did it come from?

\section{Books in brief}

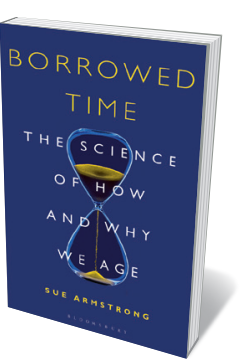

\section{Borrowed Time}

Sue Armstrong BLoOMSBuRy (2019)

Is death genetically programmed? Is ageing a disease? Engrossing questions throng science writer Sue Armstrong's round-up of research on the biology of ageing. She brings us up to speed on the illuminating (if partial) answers through work by the likes of biologist Tom Kirkwood on the 'disposable soma' theory of limited resources; Nobel laureates Elizabeth Blackburn, Carol Greider and Jack Szostak on telomeres, the end caps of chromosomes; evolutionary biologist Steven Austad on the long-lived clam Arctica islandica; and various Alzheimer's disease specialists. A rich, timely study for the era of 'global ageing'.

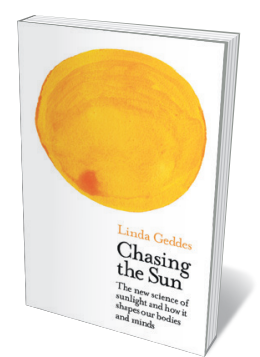

\section{Chasing the Sun}

Linda Geddes PROFILE (2019)

Light pollution, indoor lifestyles and glowing screens can play havoc with our health. So shows science writer Linda Geddes in her deft study of chronobiology, the science of organismic cycles in relation to solar rhythms. Geddes marshals an array of findings on everything from the suprachiasmatic nucleus - the cluster of cells in the brain that acts as the body's master clock — to US Amish communities living dawn to dusk and off grid, and submarine crews who rarely see the Sun. She even turns guinea pig to test measurable benefits from candlelight and outdoor exercise.

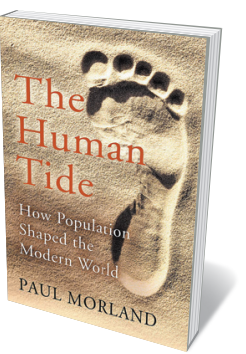

The Human Tide

Paul Morland JOHN MURRAY (2019)

The Industrial Revolution sparked a triple boom: in technology, population and emigration. In this sweeping treatise spanning the past 200 years, demographer Paul Morland traces the surge of humans in numbers and on the move, and the transformations wrought by this accelerating "demographic whirlwind" on individuals, nations and empires. It's an extraordinary ebb and flow, from the 4 million Italians who poured into the United States in the 35 years before the First World War to today's tide of African children making harrowing journeys across the Mediterranean.

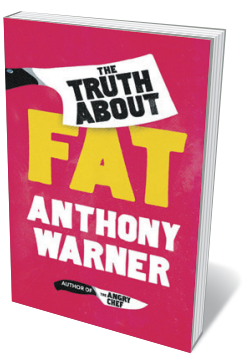

\section{The Truth About Fat}

Anthony Warner ONEWORLD (2019)

In this trenchant manifesto-cum-study, Anthony Warner (aka the Angry Chef) takes a honed knife to flabby theorizing about what makes us fat. He examines proven and putative drivers, including hormones such as leptin, calories, genetic factors, the human microbiome, inactivity, dietary fats and carbohydrates, environment and poverty. Given the complexities of the findings, Warner argues for a system-level solution involving public-health officials and communities. He also calls for an end to the moral panic surrounding fat. A nuanced approach to this global issue.

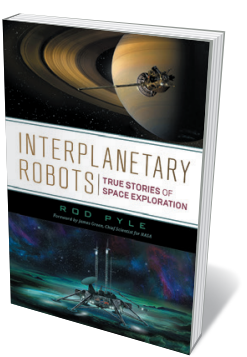

\section{Interplanetary Robots}

Rod Pyle PROMETHEUS (2019)

On 15 July 1965, NASA's Mariner 4 probe sent back grainy images of Mars, revealing a forbidding surface that destroyed florid Victorian speculation in a trice. Over the next 50 years, robotic missions researched the rest of Earth's planetary neighbours. Space writer Rod Pyle tours craft from the Soviet Luna 3, which snapped the Moon's far side in 1959, to NASA's New Horizons and its 2015 fly-by of Pluto. Sandwiched between these are tales of the Voyagers, Galileo, Cassini and more. Geeky escapism at its best. Barbara Kiser 